\title{
A Facile Route Towards Inorganic Particles with Two Distinct Compartments Based on Electro-Hydrodynamic Co-Jetting
}

\author{
Weipeng Lv, Kyung Jin Lee, Sangyeul Hwang, Tae-Hong Park, Fengbao Zhang, \\ Joerg Lahann*
}

The design and synthesis of Janus particles has been of broad interest in recent years because of their importance for both of fundamental and applied sciences. ${ }^{[1]}$ Initially, the main emphasis has been placed on controlling the surface patchiness of particles, where the anisotropy originates from asymmetrical surface functionalities. ${ }^{[2]}$ More recently, multicompartmental particles have emerged that feature compositionally distinct pockets of distinct materials..$^{[3]}$ The multicompartmental architecture not only gives rise to defined surface patterns, but can result in distinct bulk compartments and therefore truly anisotropic functionalities. Although a number of methods to control surface patchiness, such as line-of-side deposition of metals, or selective surface modification, are similarly applicable to organic as well as inorganic particles, very little is known about bulk-compartmentalized inorganic micro- and nanoparticles. ${ }^{[2 \mathrm{~b}, 4]}$ Preparation of purely inorganic particles with defined compartmentalization has been more challenging, and their preparation has been limited to toposelective surface modification, ${ }^{[5]}$ template-directed self-assembly, ${ }^{[6]}$ controlled phase separation, ${ }^{[7]}$ and controlled surface nucleation. ${ }^{[8]}$ Impressive particles including dumbbell-, snowman- or acorn-like particles have been prepared based on these methods to date.

Herein, we report a novel approach towards compartmentalized inorganic microparticles using EHD co-jetting with mixtures of inorganic precursors and organic polymers followed

\author{
Dr. W. Lv, Dr. K. J. Lee, Dr. S. Hwang, \\ Dr. T.-H. Park, Prof. J. Lahann \\ Department of Chemical Engineering \\ University of Michigan \\ Ann Arbor, MI, 48109, USA \\ E-mail: lahann@umich.edu \\ Dr. W. Lv, Prof. F. Zhang \\ School of Chemical Engineering and Technology \\ Tianjin University \\ Tianjin, 300072, China \\ Prof. J. Lahann \\ Department of Macromolecular Science and Engineering \\ University of Michigan \\ Ann Arbor, MI, 48109, USA \\ Prof. J. Lahann \\ Biomedical Engineering \\ University of Michigan \\ Ann Arbor, MI, 48109, USA \\ Prof. J. Lahann \\ Materials Science and Engineering \\ University of Michigan \\ Ann Arbor, MI, 48109, USA
}

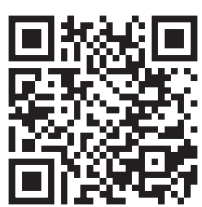

DOI: 10.1002/ppsc.201300123 by calcination to convert the composite particles into stable inorganic building blocks with defined bulk anisotropy. EHD co-jetting, which has been initially developed for soft matter applications, has been successfully used for spherical as well as non-spherical particles. These Janus-type particles were composed of a range of different polymers as well as selected polymer/nanocrystal composites. ${ }^{[2 a, 9]}$ Here, we attempt to adopt this technology to create compartmentalized inorganic micronand nanoscale Janus particles fully composed of inorganic materials.

The experimental setup for EHD co-jetting of bicompartmental microparticles is depicted in Figure 1A. To establish suitable process parameters, two solutions containing poly(lactic-co-glycolic acid) (PLGA) of $3.0 \mathrm{w} / \mathrm{v} \%$ and inorganic precursor of $3 \mathrm{v} / \mathrm{v} \%$ such as tetraethyl orthosilicate (TEOS) and titanium tetrabutoxide (TTBO) were delivered to each nozzle. A mixture of chloroform and dimethylformamide (DMF) in 97:3 volume ratios was used as the solvent for jetting. Electrohydrodynamic jetting of these solutions resulted in solid particles. To visualize the individual compartments of the particles, one of the jetting solutions was loaded with blue fluorescent dye (poly[2-methoxy-5-(2-ethylhexyloxy)-1,4-phenylene vinylene] (MEHPV), $0.05 \mathrm{w} / \mathrm{v} \%$ ), while the other one contained green fluorescent dye (poly[tris(2,5-bis(hexyloxy)-1,4-phenylenevinylene)] (PTDPV), $0.05 \mathrm{w} / \mathrm{v} \%)$. Both solutions were pumped through the capillary needles with a flow rate of $0.20 \mathrm{~mL} \mathrm{~h}^{-1}$ for each. The liquid droplet at the end of the capillaries was converted to a single Taylor cone by applying a DC voltage of $6-7 \mathrm{kV}$ to the capillaries. The formation of an electrified jet leads to the evaporation of volatile solvents and nanoprecipitation of the nonvolatile PLGA and TEOS compounds. A quartz plate was used as collecting substrate placed on conductive Al foil.

Fundamentally, EHD co-jetting relies on the laminar flow of two or more miscible polymer solutions under an electric field, which results in the distortion of the droplet at the outlet into a Taylor cone. Recent work has demonstrated the feasibility of processing nanoparticle suspensions with this method. [9d,e] When extending the application of EHD co-jetting to the engineering of inorganic particles with multiple compartments, it is imperative that stable Taylor cones are formed. In practice, this has constituted a major challenge and hampered expanding this technique beyond polymeric materials so far. It is well known that the formation of Taylor cones is aided by jetting solutions with high, yet controlled viscosities. To increase the viscosity of the jetting solutions containing inorganic precursors, i.e., TEOS, partially crosslinked precursors were typically processed in the past. ${ }^{[10]}$ This approach has two fundamental shortcomings: i) it 


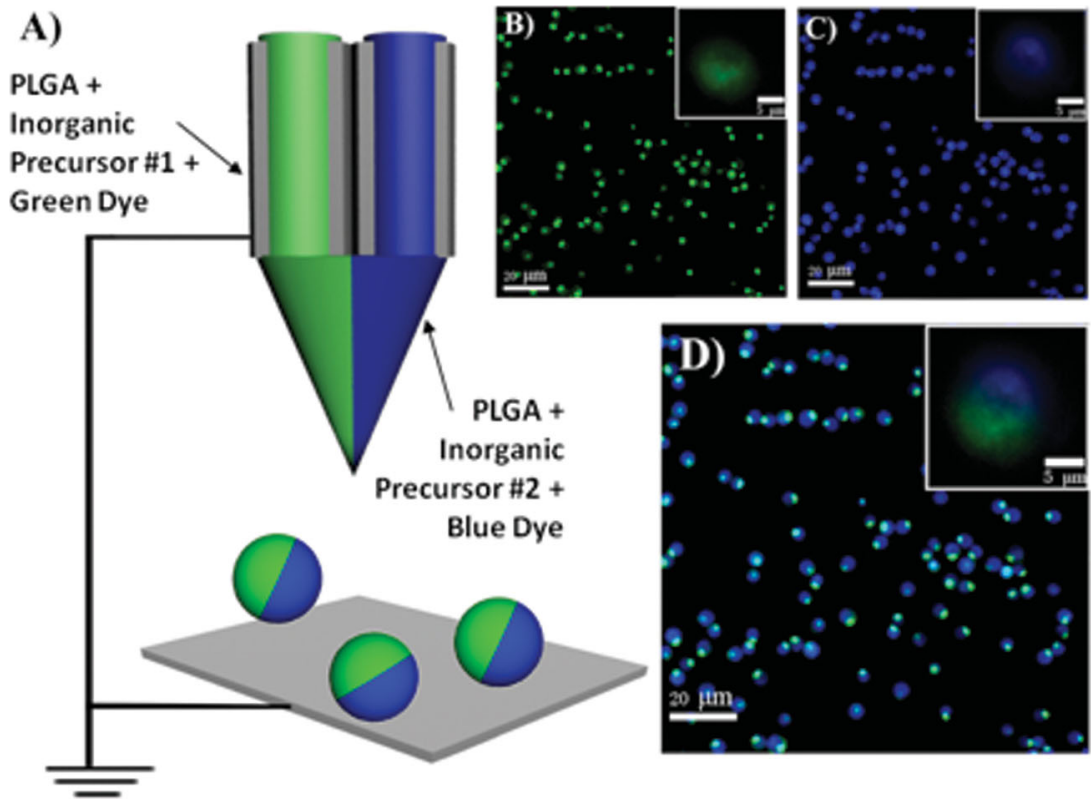

Figure 1. A) Schematic illustration of the electrohydrodynamic co-jetting process used for the synthesis of bicompartmental Janus composite particles. B) Green, C) blue, and D) overlay images were obtained by confocal laser scanning microscopy (CLSM) of the bicompartmental particles; insets show high-resolution images.

requires processing of a reacting system that rapidly changes the state of solution with time. This aspect creates inhomogenities over time, limits the applicability, and can only be used to create fibers because of their high viscosity (for particles, jetting solutions with somewhat lower viscosities is required). ii) To induce gelation, the precursors were processed in water or water compatible solvents, which greatly limit the materials selection.

Consequently, we focused our efforts on developing approached that utilize water-free solvents. The anhydrous conditions prevent uncontrolled gelation and allow for modulation of the viscosities of the jetting solutions through polymer additives. Based on our initial experiments, spherical particles can be obtained by EHD co-jetting under anhydrous conditions, as shown in conforcal laser scanning microscopy image of the as-jetted PLGA/TEOS composite particles (Figure 1B-D). The green (Figure 1B) and blue color (Figure 1C) in the microscopy images represent the selectively compartmentalized PTDPV and MEHPV polymers that were collocated with the inorganic precursors. The overlay image (Figure 1D) of two fluorescence channels reveals a clear interface between the compartments confirming the formation of bicompartmental composite particles under the conditions of EHD co-jetting. These findings confirm the successful preparation of Janus-type particles composed of a polymer matrix containing inorganic precursor.

SEM images at different magnifications were used to characterize the surface morphology and size distributions of the PLGA/TEOS composite particles. Here, we added magnetic nanoparticles (30 nm, in diameter) in one side to visualize compositional anisotropy. The whole particles were found to be perfectly spherical with an average diameter of $5.5 \pm 1.0 \mu \mathrm{m}$ (Figure 2A). The high-resolution SEM images in Figure 2B further confirm the selective encapsulation of magnetite nanocrystals in one compartment. This compartment has a rougher surface compared with the smooth surface of the magnetitefree compartment. Since EHD co-jetting of PLGA-based polymers has been proved to be suitable for easy and precise control over particles anisotropy, shape, and size, ${ }^{[9 \mathrm{~b}]}$ the use of PLGA as the polymer template was explored based on the expectation that it will lead to uniform spherical particles with welldefined and distinct compartments.

In addition to employing anhydrous conditions, no acid or base catalyst was added to the jetting solutions during the jetting procedure. In contrast to previous strategies, ${ }^{[10]}$ we thus were able to conduct non-reactive electrospraying of silica precursors under fully anhydrous conditions. We hypothesized that the non-reactive jetting approach will provide several advantages in terms of material's flexibility, scalability, and reproducibility over the conventional reactive jetting methodology.

After establishing that the inorganic precursors can be successfully processed along with the PLGA polymer, we next focused on the removal of the polymer matrix to obtain neat inorganic particles. In this step, it was critical to maintain both shape and bicompartmental character during conversion of the composite particles into inorganic particles. As-jetted PLGA/TEOS composite particles were treated with $\mathrm{HCl}$ and water vapor, which simultaneously served as a catalyst for crosslinking and hydrolysis agent. Using the vapor treatment prior to calcination ensured formation of a layer of $\mathrm{SiO}_{2}$ on the particle surface and was critical for avoiding interparticle crosslinking. In addition, without the vapor treatment, cross-linking of TEOS was incomplete and only small $\mathrm{SiO}_{2}$ grains rather than spherical particles were obtained during calcination. In contrast, calcination of composite particles pretreated with $\mathrm{HCl}$ and water vapor at $500{ }^{\circ} \mathrm{C}$ for $4 \mathrm{~h}$ yielded neat $\mathrm{SiO}_{2}$ particles. During the calcination step, which was conducted at elevated temperatures of $500{ }^{\circ} \mathrm{C}$, condensation of the silica precursors occurred and the PLGA polymer matrix was simultaneously removed. This is consistent with the Fourier transform
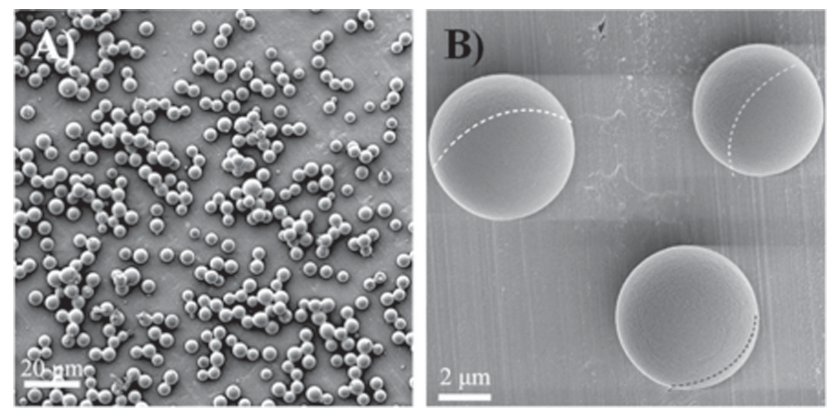

Figure 2. A,B) Typical SEM images of bicompartmental $\mathrm{SiO}_{2}$ particles with different magnifications show monodisperse and perfectly spherical particles with well-defined interfaces between different surface morphologies. 


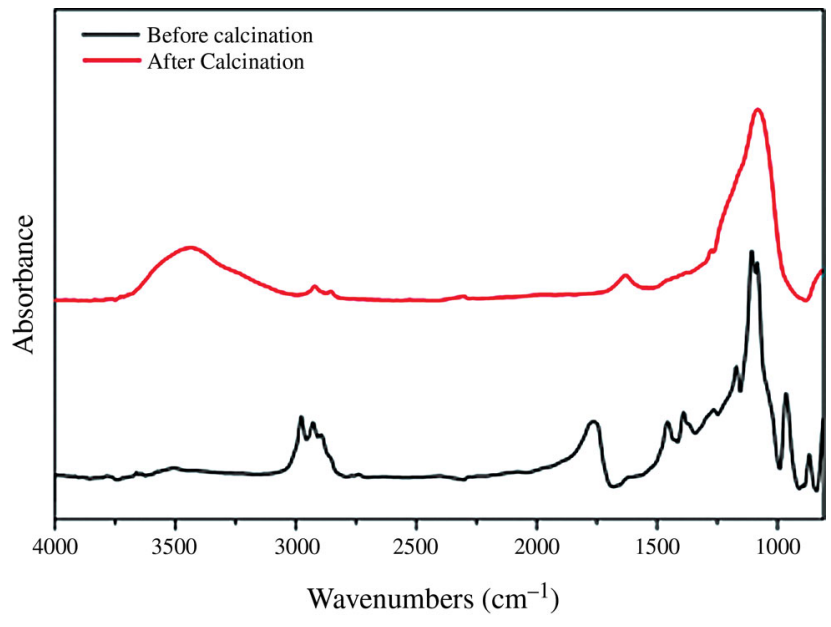

Figure 3. FTIR spectra of bicompartmental $\mathrm{SiO}_{2}$ particles before and after calcination.

infrared (FTIR) spectra of bicompartmental particles before and after calcination (Figure 3). The FTIR spectrum before calcination shows the characteristic bands for PLGA such as $\mathrm{C}-\mathrm{H}$ and $\mathrm{C}=\mathrm{O}$ stretching at $2800-3000$ and $1758 \mathrm{~cm}^{-1}$, respectively. After calcination, the characteristic PLGA bands disappeared and an asymmetric $\mathrm{Si}-\mathrm{O}-\mathrm{Si}$ stretching band emerged at $1082 \mathrm{~cm}^{-1}$. Unlike previous studies using thoroughly or partially hydrolyzed TEOS sol-gel as solutions for electrojetting of $\mathrm{SiO}_{2}$ fibers, ${ }^{[10]}$ the TEOS polymerization was decoupled from the particle formation process, resulting in successful preparation of inorganic particles (not the fibrous structure) using electrospraying.

After successful removal of PLGA and the preparation of $\mathrm{SiO}_{2}$ particles, we verified that spherical shape and bicompartmental character were retained. SEM analysis was used to investigate the shape and surface morphology of the final $\mathrm{SiO}_{2}$ particles, as shown in Figure 4A. During the vapor treatment of the original composite particles, a protective shell composed of small-size $\mathrm{SiO}_{2}$ grains was formed by pre-crosslinking of TEOS. Therefore, the condensation of $\mathrm{SiO}_{2}$ particles is restricted to individual particles and the spherical shape of the bicompartmental particles is maintained in the subsequent calcination step. In addition, the particles were found to shrink after calcination, as indicated by a decreased average particle diameter of $4.0 \pm 1.5 \mu \mathrm{m}$ as compared with $5.5 \pm 1.0 \mu \mathrm{m}$ before calcination. The particle shrinkage during calcination can be ascribed to the solidification of $\mathrm{SiO}_{2}$ by thermal treatment, and the removal of the PLGA matrix.

To further confirm the condensation reaction and to investigate the bicompartmental architecture of the inorganic particles, we employed 2D EDX element distribution mapping. In this case, the iron oxide nanocrystals compartmentalized in one side served as visualization tracers. EDX maps representing Fe and Si distributions are shown in Figure 4B,C. Both elemental maps were measured at the same place, as shown in Figure 4A. The clear interface shown in the Fe/Si overlap image (Figure 4D) confirms the bicompartmental nature of $\mathrm{SiO}_{2}$ particle. One hemisphere of the particle contains both $\mathrm{SiO}_{2}$ and $\mathrm{Fe}_{2} \mathrm{O}_{3}$ while the other one contains only $\mathrm{SiO}_{2}$.
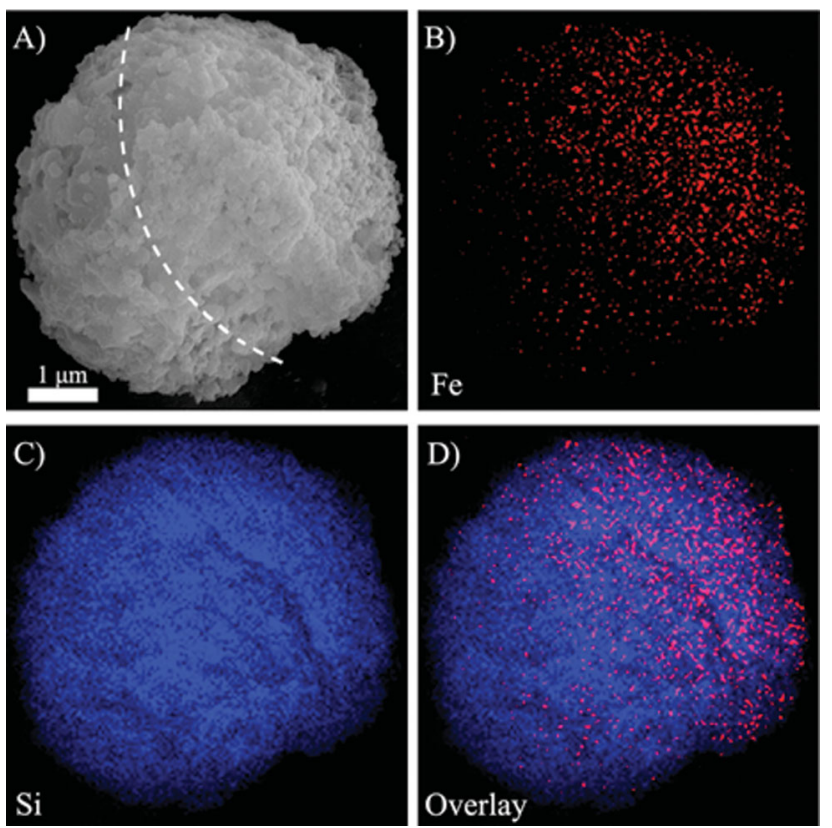

Figure 4. Typical SEM images and EDX mapping of bicompartmental $\mathrm{SiO}_{2}$ particles after calcination. A) SEM image, B) Fe distribution, C) Si distribution, and D) Fe/Si overlay of the images for an individual particle. The two-dimensional mapping of element distribution has been measured by energy dispersive X-ray spectroscopy (EDX).

The ultimate goal of this study was to explore the possibility of producing bicompartmental particles with compartments made of distinct inorganic materials. Using an EHD co-jetting arrangement similar to the one described above, composite particles containing different inorganic precursors in two compartments were prepared (TEOS for $\mathrm{SiO}_{2}$ and TTBO for $\mathrm{TiO}_{2}$ ). Specifically, two jetting solutions were used: 1) $3.0 \mathrm{w} / \mathrm{v} \%$ PLGA and $2.3 \mathrm{w} / \mathrm{v} \%$ TEOS; and 2) $3.0 \mathrm{w} / \mathrm{v} \%$ PLGA and $3.7 \mathrm{w} / \mathrm{v} \%$ TTBO, in $97: 3$ volume ratios of chloroform and DMF. In this case, the flow rates and the jetting voltage were controlled at $0.30 \mathrm{~mL} \mathrm{~h}^{-1}$ and $7-8 \mathrm{kV}$, respectively. A typical SEM image of the as-jetted particles is shown in Figure 5A. It confirms that most of the particles have near-spherical shapes with an average diameter of $600 \pm 300 \mathrm{~nm}$. After PLGA removal and TEOS/TTBO condensation due to calcination at $500{ }^{\circ} \mathrm{C}$ for $4 \mathrm{~h}$, the particle shapes were maintained and the average diameter decreased to $400 \pm 200 \mathrm{~nm}$ (Figure 5B). In the case of submicrometer-sized composite particles, $\mathrm{HCl}$ treatment is not needed to obtain complete crosslinking of the inorganic precursors. As shown in the high-resolution SEM images (Figure 5C), the $\mathrm{SiO}_{2}$ and $\mathrm{TiO}_{2}$ compartments, hierarchical structures similar to the ones shown in Figure 4A for neat $\mathrm{SiO}_{2}$ were obtained. The second hemisphere displayed a rather smooth surface - separated by a clear interface between the two compartments. To unambiguously confirm that particles with distinct titania and silica compartments were formed, we attempted to selectively remove the $\mathrm{TiO}_{2}$ compartment by chemical etching. Figure 5D shows the SEM image and EDX spectrum of the bicompartmental particles after chemical etching with $\mathrm{HCl}$. Indeed, half of the particles were etched away and well-defined half-shells were 

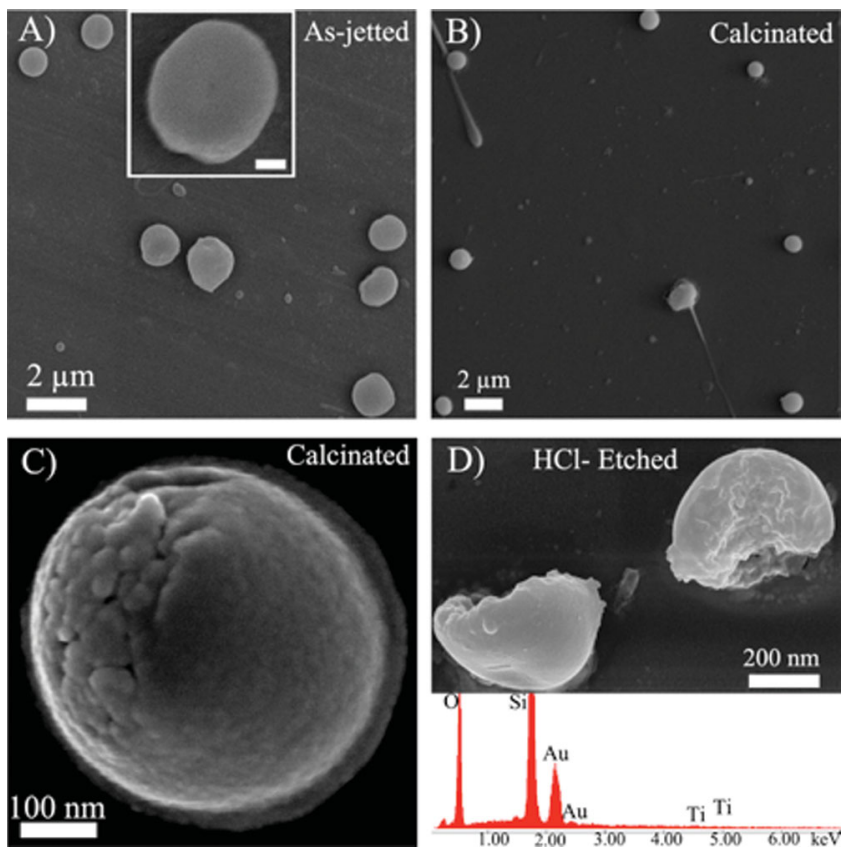

Figure 5. A) Typical SEM images of as-jetted particles with different inorganic precursors (TEOS and TTBO) (the inset scale bar: $100 \mathrm{~nm}$ ). $B, C)$ SEM images at different magnifications of calcinated particles. D) SEM image of calcinated particles after selective removing of $\mathrm{TiO}_{2}$ compartment by $\mathrm{HCl}$ etching showing the hemisphere of $\mathrm{SiO}_{2}$ compartment. The complete removal of $\mathrm{TiO}_{2}$ is confirmed by EDX spectrum (lower panel).

obtained. The absence of the Ti signal in the EDX spectrum of the remaining half-shells confirms that the $\mathrm{TiO}_{2}$ compartment was completely removed (lower panel in Figure 5D). Taken together, these data confirm successful preparation of compositionally different inorganic particles by EHD co-jetting.

In summary, bicompartmental $\mathrm{SiO}_{2}$ particles with compositionally different compartments were prepared by non-reactive EHD co-jetting of $\mathrm{SiO}_{2}$ precursor (TEOS) in water-free solvent. The stable jetting was facilitated by addition of PLGA in the jetting solutions, which was used as a sacrificing polymer matrix. A subsequent calcination step ensured simultaneous condensation of the inorganic precursors and PLGA removal. After calcination, the shape and bicompartmental character of the particles were retained. Finally, particles with distinct compartments made up of different materials $\left(\mathrm{TiO}_{2}\right.$ and $\left.\mathrm{SiO}_{2}\right)$ were successfully prepared by EHD co-jetting process. Subsequent calcination yielded novel inorganic Janus particles. The herein reported methodology is sufficiently robust and versatile to extend the application of the EHD co-jetting technique from soft matter particles to compartmentalized inorganic particles. Future refinements of the EHD co-jetting methodology may result in novel types of multicompartmental inorganic particles with defined shapes, sizes, anisotropy, and compositions. However, such progress will require systematic evaluation of process parameters and inorganic precursor chemistries. As these studies progress, novel multicompartmental materials may be prepared that will ultimately find practical applications in catalysis, display technologies, or energy research.

Received: March 9, 2013 Revised: May 1, 2013 Published online: June 25, 2013

[1] a) A. Perro, S. Reculusa, S. Ravaine, E. Bourgeat-Lami, E. Duguet, J. Mater. Chem. 2005, 15, 3745; b) D. Rodriguez-Fernandez, L. Liz-Marzan, Part. Part. Syst. Charact. 2013, 30, 46; c) S. Mitragotri, J. Lahann, Nat. Mater. 2009, 8, 15; d) K. J. Lee, J. Yoon, J. Lahann, Curr. Opin. Colloid Interface Sci. 2011, 16, 195; e) J. Yoon, K. J. Lee, J. Lahann, J. Mater. Chem. 2011, 21, 8502; f) A. Walther, A. H. E. Muller, Soft Matter 2008, 4, 663; g) Q. Chen, J. K. Whitmer, S. Jiang, S. C. Bae, E. Luijten, S. Granick, Science 2011, 331, 199.

[2] a) K. H. Roh, D. C. Martin, J. Lahann, Nat. Mater. 2005, 4, 759; b) A. B. Pawar, I. Kretzschmar, Macromol. Rapid Commun. 2010, 31, 150; c) D. Dendukuri, P. S. Doyle, Adv. Mater. 2009, 21, 4071; d) W. Lv, Kyung K. Lee, J. Li, T.-H. Park, S. Hwang, A. J. Hart, F. Zhang, J. Lahann, Small 2012, 8, 3116.

[3] J. Lahann, Small 2011, 7, 1149.

[4] a) X. Y. Ling, I. Y. Phang, C. Acikgoz, M. D. Yilmaz, M. A. Hempenius, G. J. Vancso, J. Huskens, Angew. Chem. Int. Ed. 2009, 48, 7677; b) K. D. Anderson, M. Luo, R. Jakubiak, R. R. Naik, T. J. Bunning, V. V. Tsukruk, Chem. Mater. 2010, 22, 3259; c) S. Jiang, M. J. Schultz, Q. Chen, J. S. Moore, S. Granick, Langmuir 2008, 24, 10073.

[5] a) V. N. Paunov, O. J. Cayre, Adv. Mater. 2004, 16, 788; b) Y. Lu, H. Xiong, X. Jiang, Y. Xia, M. Prentiss, G. M. Whitesides, J. Am. Chem. Soc. 2003, 125, 12724; c) H. Gu, Z. Yang, J. Gao, C. K. Chang, B. Xu, J. Am. Chem. Soc. 2004, 127, 34.

[6] a) Y. Yin, Y. Lu, Y. Xia, J. Am. Chem. Soc. 2001, 123, 771; b) Y. Xia, Y. Yin, Y. Lu, J. McLellan, Adv. Funct. Mater. 2003, 13, 907.

[7] a) H. Gu, R. Zheng, X. Zhang, B. Xu, J. Am. Chem. Soc. 2004, 126, 5664; b) P. Mulvaney, M. Giersig, T. Ung, L. M. Liz-Marzán, Adv. Mater. 1997, 9, 570.

[8] a) T. Teranishi, Y. Inoue, M. Nakaya, Y. Oumi, T. Sano, J. Am. Chem. Soc. 2004, 126, 9914; b) H. Yu, M. Chen, P. M. Rice, S. X. Wang, R. L. White, S. Sun, Nano Lett. 2005, 5, 379.

[9] a) K. H. Roh, D. C. Martin, J. Lahann, J. Am. Chem. Soc. 2006, 128, 6796; b) S. Bhaskar, K. M. Pollock, M. Yoshida, J. Lahann, Small 2010, 6, 404; c) S. Bhaskar, J. Hitt, S. W. L. Chang, J. Lahann, Angew. Chem. Int. Ed. 2009, 48, 4589; d) D. W. Lim, S. Hwang, O. Uzun, F. Stellacci, J. Lahann, Macromol. Rapid Commun. 2010, 31, 176; e) S. Hwang, K. H. Roh, D. W. Lim, G. Y. Wang, C. Uher, J. Lahann, Phys. Chem. Chem. Phys. 2010, 12, 11894.

[10] a) S. J. Wu, F. T. Li, Y. N. Wu, R. Xu, G. T. Li, Chem. Commun. 2010, 46, 1694; b) S. H. Zhan, D. R. Chen, X. L. Jiao, Y. Song, Chem. Commun. 2007, 2043; c) W. Wang, J. Y. Zhou, S. S. Zhang, J. Song, H. G. Duan, M. Zhou, C. S. Gong, Z. Bao, B. A. Lu, X. D. Li, W. Lan, E. Q. Xie, J. Mater. Chem. 2010, 20, 9068. 